\title{
Vulvar malignant melanoma: a rare occurrence in a young woman
}

\begin{abstract}
Malignant melanoma is one of the most belligerent neoplasm which arises from the skin melanocytes. The occurrence of this tumor in the female genital tract system is quite uncommon, where it usually involves the elderly women. It is important to recognize and give comprehensive diagnosis of such an extracutaneous melanoma, as it has poor prognosis in comparison to the cutaneous melanoma and requires different therapeutic approach. Herein, we describe a rare case of vulvar malignant melanoma in a young nulliparous female, which is not the usual age group for its presentation.
\end{abstract}

Keywords: melanoma, vulva, young adult, prognosis
Volume II Issue 4 - 2020

\author{
Sonam Sharma,' Shipra Singhal, ${ }^{2}$ Rajat \\ $\mathrm{Kamra}^{3}$ \\ 'Department of Pathology, Kalpana Chawla Government \\ Medical College, Karnal, Haryana, India \\ 2Department of Pathology, Vardhman Mahavir Medical College \& \\ Safdarjung Hospital, New Delhi, India \\ ${ }^{3}$ Department of Oncosurgery, Vardhman Mahavir Medical \\ College \& Safdarjung Hospital, New Delhi, India
}

Correspondence: Sonam Sharma, Department of Pathology, Kalpana Chawla Government Medical College, Karnal, Haryana, India, Tel 999984I393, Email drsonamsharma@gmail.com

Received: July 02, 2020 | Published: July 202020

\section{Introduction}

Vulvar cancers are rare, accounting for 3-5\% of all the gynecological malignancies and less than $1 \%$ of all malignant neoplasms in women. Its estimated incidence is $1-2$ cases per 100,000 women per year. Squamous cell carcinoma is the most common histological type, constituting for $90 \%$ of the invasive vulvar cancers followed by the malignant melanoma (5-6\%). Sarcoma, basal cell carcinoma, adenocarcinoma and vulvar Paget's disease account for less than $2 \%$ of the cases. ${ }^{2}$ Nevertheless, amongst all, vulvar malignant melanoma is an extremely challenging neoplasm as it shares its property with other extracutaneous melanomas which emerge in the sun-shielded areas of the body. These melanomas often cause serious therapeutic problems either by evading an early detection and thus, advancing to a considerable size before their diagnosis is made or because of their extreme biological aggressiveness. We present one such uncommon case of vulvar malignant melanoma in a young nulliparous female so as to create awareness among the clinicians about this perplexing entity.

\section{Case report}

A 24-year-old unmarried, nulliparous female presented to the surgery outpatient department with a vulvar region mass of 4 months duration. It was associated with persistent itching and an occasional episode of bleeding. She also revealed that the mass was initially small but since the past 2 months it was gradually increasing in its size. There was no history of fever, loss of weight/appetite or any bowel or bladder symptoms. Her medical, past as well as her family history of any cancer was non-contributory. She had attained menarche at the age of 13 years and had regular cycles with an average flow. On general physical examination, she was thin built and anemic. Her vitals were within the normal limits. No lymphadenopathy was evident. Per abdomen examination revealed soft and non-tender abdomen with no evidence of ascites or any organomegaly. On local examination, a nodular, grey to black color growth of $5 \mathrm{~cm} \times 2 \mathrm{~cm}$ size arising from the left labia majora was seen. The surface of it was ulcerated and its margins were irregular. However, it was not encroaching onto the structures like urethra and vagina. Per speculum examination was unremarkable. Bimanual pelvic examination revealed anteverted nulliparous sized uterus with free bilateral fornices and without palpable adnexal masses. On per rectal examination, rectal mucosa was free. All other systemic examinations did not show any abnormality. Her routine hematological investigations exhibited microcytic hypochromic blood picture. Urine and blood cultures were negative. Kidney and liver function tests were normal. Serum antibodies to human immunodeficiency virus, hepatitis B surface antigen, and syphilis were negative. X-ray chest was normal. A whole body scan with the help of Positron emission tomographycomputed tomography (PET-CT) was performed, which showed fluorodeoxyglucose uptake in the vulva only. There was no evidence of any local or distant spread. Based on these clinical and radiological findings, a vulvar malignancy was suspected which was clinically staged as International Federation of Gynecology and Obstetrics (FIGO) stage 1B. A wide local excision of the vulvar mass was done along with an ipsilateral superficial inguinal lymphadenectomy and the specimens were sent for histopathological examination in two different containers. Grossly, a skin flap measuring $6 \mathrm{~cm} \times 4 \mathrm{~cm} \times$ $3 \mathrm{~cm}$ in size was received in one container. An irregular, pigmented growth was seen on the centre of the flap. On serial sectioning of the growth, a grey white tumor measuring $4.5 \mathrm{~cm} \times 2 \mathrm{~cm} \times 2 \mathrm{~cm}$ in size was seen. All the skin margins were free of tumor. In another container, a fibro fatty soft tissue piece measuring $5 \mathrm{~cm} \times 3 \mathrm{~cm} \times 2 \mathrm{~cm}$ in size was received. On cut, eight lymph nodes were resected out. Microscopic examination showed keratinized stratified squamous epithelium which was ulcerated at places. The subepithelial tissue revealed tumor cells which were arranged in the form of nests, cords and sheets. The cells exhibited variation in shape ranging from round to spindle. The nuclei were round to oval to spindle in shape with presence of clumped chromatin and prominent nucleoli. There was presence of brisk mitotic activity including atypical mitotic figures. Few of these cells contained intracytoplasmic melanin pigment (Figure 1). All the resected margins and lymph nodes were found to be free of tumor. On immunohistochemistry (IHC), the tumor cells were diffusely and strongly positive for Human Melanoma Black (HMB-45) and S-100 
(Figure 2). Based on these histological and immunohistochemical findings, a final diagnosis of vulvar malignant melanoma with Clark's level IV was made. The postoperative period of the patient was uneventful. She is under regular monthly follow-up and has no signs or symptoms of any recurrence or metastasis so far.



Figure I Vulvar epithelium with underlying tumor cells containing fine granular intracytoplasmic melanin pigment $(\mathrm{H}$ and $\mathrm{E}, \mathrm{xI00})$.

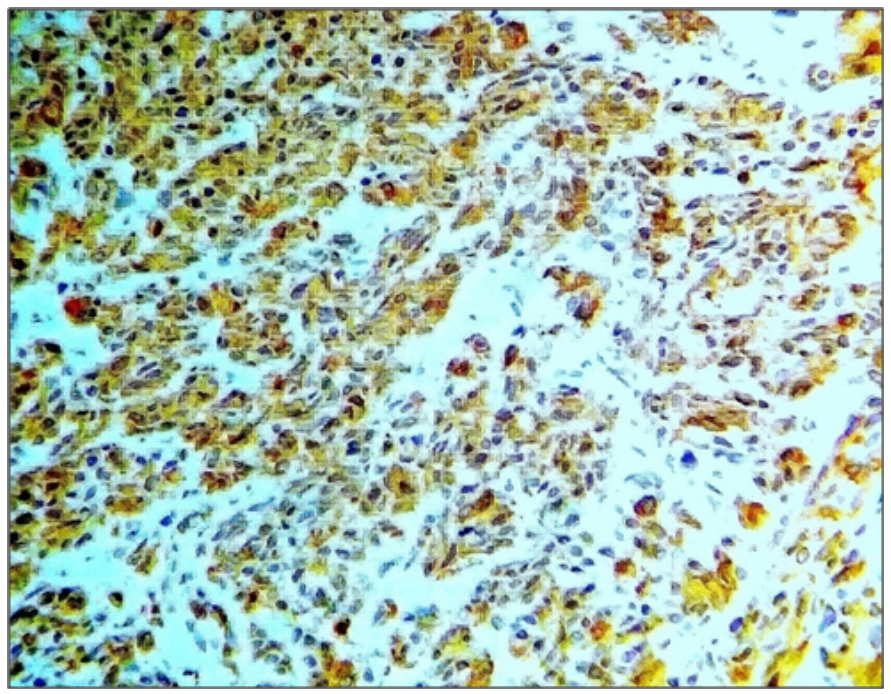

Figure 2 Tumor cells showing positive HMB-45 immunoexpression (IHC, x200).

\section{Discussion}

Primary malignant melanoma of the female genital tract system is rare, accounting for 3\% of all the malignant melanomas and $18 \%$ of mucosal melanomas. It most commonly arises in the vulva, followed by the vagina, uterus, ovaries and cervix. ${ }^{3}$ Despite its rarity, vulvar malignant melanoma is the second most common vulvar malignancy after squamous cell carcinoma and accounts for $8-10 \%$ of all the malignant tumors involving the vulva. ${ }^{4}$ Vulvar malignant melanomas, although involve the hairy and glabrous skin of the vulva but are still considered as one of the mucosal or extracutaneous melanomas rather than melanomas of the skin, because of their sun-shielded location and continuity with the vaginal mucosa. Various clinicopathological features of this rare entity warrant an attention for its timely detection and therapeutic intervention. Clinically, it occurs predominantly in older women, with a very tall peak in the sixth and seventh decades of life with approximately $90 \%$ of the cases affecting the white race. ${ }^{5}$ The labia majora is the most common location of this tumor $(52 \%)$ followed by the labia minora $(18 \%)$, clitoris $(10-15 \%)$ and bartholin's glands (1-3\%). The signs and symptoms of patients with this tumor are initially non-specific, however, most of them present with an asymptomatic vulvar pigmented or non-pigmented lesion and occasionally with vaginal bleeding and/or discharge, pruritus, pain, and urination discomfort. ${ }^{6}$ It can be categorized according to clinical and histological parameters in order of their incidence into superficial spreading melanoma (40-56\%), mucosal lentiginous (27$57 \%)$, nodular melanoma (22-28\%), and unclassified (12-16\%). The present case had similar clinical presentation of a pigmented growth of the labia majora, however, the young age of the patient was unlike that is usually seen to be involved by this tumor.

The pathogenesis and risk factors for vulvar malignant melanoma is still a subject of speculations as although the etiological role of ultraviolet (UV) radiation has been well-documented for cutaneous malignant melanomas arising in sun-exposed areas but this theory fails to explain the occurrence of melanoma in sun-shielded areas like the vulva. Nevertheless, it has been mentioned that the UV radiation may still be indirectly involved by causing a cell-mediated systemic alteration of the immunity which can create a more favorable environment for the development of this vulvar malignancy. ${ }^{7}$ On the other hand, it has also been assumed that these UV radiation have a protective effect against this malignancy, possibly owing to its role in vitamin D photosynthesis in the sun-exposed skin. ${ }^{8}$ Therefore, some researchers have suggested that the vulvar malignant melanoma exemplifies the possible product of etiological factors other than UV radiation and these factors can be tissue specific or can be derived from environmental toxins, drugs, or viruses such as human papilloma viruses, human herpes viruses, and polyomavirus. ${ }^{6,9,10}$ This fact has also been well-supported by some authors who have observed that the vulvar malignant melanoma usually arises de novo especially in those which develop on the glabrous skin and are more aggressive whereas a pre-existing junctional or compound nevi can be considered a precursor of melanoma arising from the vulvar hairy skin. Thereby, emphasizing that the anatomical site and local tissue factors rather than the histogenetic type defines a distinct type of melanoma. ${ }^{11}$ Many molecular mechanisms have been additionally proposed to play a crucial role. These include mutations of RAS oncogenes, p53 tumor suppressor gene, c-KIT and BRAF protooncogenes. Multiple chromosomal aberrations, most commonly in chromosome 1, especially 1q12-q41 and of chromosome $6 p$, have also been implicated in this tumorigenesis.

The diagnosis of vulvar malignant melanoma directs its staging, treatment and prognosis. Therefore, early detection and proper treatment of primary as well as metastatic malignant melanoma play a substantial role in survival of the patient. ${ }^{12}$ When the primary vulvar malignant melanoma is clinically suspected, histopathology of the excised specimen renders the diagnostic as well as prognostic information. However, histopathology needs to be confirmed by using various immunostains, since a routine hematoxylin and eosin examination may lead to a differential diagnosis of poorly differentiated carcinoma. Among the various immunohistochemical markers, usually two of the following immunoperoxidase markers (i.e., S-100, KBA.62, HMB-45, Tyrosinase, MITF or Melan-A) are 
required to be positive for confirming its diagnosis. Nevertheless, histopathology also helps in staging of vulvar malignant melanomas as the Breslow, Clark and Chung staging systems reflect a better correlation with the prognosis of such patients. In addition, electron microscopy significantly contributes to its diagnosis, especially in cases where IHC is non-contributory, by an ultrastructural demonstration of melanosomes which is a conclusive feature of malignant melanoma. However, in situations where the primary is unsuspected/ unknown, Fine needle aspiration cytology may be requisitioned as a primary modality, especially if the presentation is in the form of lymph node metastasis. ${ }^{13}$ Malignant melanoma spreads in three different ways: by giving metastasis to regional lymph nodes, by direct distant metastasis, and by satellite or in-transit metastasis. In vulvar malignant melanoma, metastasis is most commonly found in the inguinal lymphatic nodes. Other reported metastatic sites are the lungs, vagina, uterus, ovaries, liver, brain, myocardium, kidneys, adrenals and stomach. Therefore, it is extremely important to evaluate the entire genital system in vulvar malignant melanoma patients. ${ }^{14}$ In such circumstances, imaging such as PET-CT is quite helpful in determining the extent of metastatic disease. In the present case, PETCT was beneficial, however, the final diagnosis was only made on the basis of histopathological and immunohistochemical findings of the resected specimen.

Although there are several treatment options for vulvar malignant melanoma, an appropriate and effective treatment protocol is yet to be defined. In most of the patients, surgery still remains the primary treatment of choice. The spectrum of surgery ranges from conservative (wide local excision of the tumor), hemi/partial/total vulvectomy to radical vulvectomy and lymph node dissection, which can either be therapeutic or elective (sentinel node biopsy/prophylactic), unilateral or bilateral, superficial and/or deep or combinations thereof. ${ }^{11}$ Nevertheless, historically recommended treatment for it has been radical vulvectomy with bilateral inguino-femoral lymphadenectomy, regardless of lesion size, thickness, or depth of invasion. ${ }^{15}$ But recently, this has become a questionable and an obsolete treatment approach as many studies have concluded that the radical surgery is disfiguring and is associated with severe morbidity as well as a high risk of local progression. Also, there is no significant improvement in the survival of patients with an early disease treated with radical surgery. ${ }^{3}$ Therefore, conservative treatment with a wide local excision has turned out to be as effective as vulvectomy for tumor-specific survival. Researchers have recommended that for the treatment of vulva malignant melanomas with thin lesions $(<1 \mathrm{~mm})$, a wide local excision with a safety distance of $1 \mathrm{~cm}$ and, with deeper lesions, an en bloc resection with safety distance of $2-3 \mathrm{~cm}$ with regional (inguinofemoral) lymphadenectomy should be done. ${ }^{16}$ Adjuvant treatments with radiotherapy, chemotherapy and immunotherapy have been looked upon in specific cases especially of local recurrences and distant metastasis however; there have been no additional survival benefits with these modalities. Nevertheless, with the discovery of cKIT and BRAF mutations in vulvar malignant melanoma, development of targeted agents that inhibit these oncogenic pathways can lead to significant advances in the treatment of metastatic malignant melanomas.

The prognosis of vulvar malignant melanoma patients is poor, irrespective of any treatment because of the diffuse and rich vascular and lymphatic plexus of vulva as well as most of the patients are diagnosed at advanced stage owing to the delayed diagnosis. Both these factors contribute to an early tumor spread and development of metastasis. Nevertheless, the main prognostic factors are demographic characteristics, tumor localization, presence of groin node metastasis, and various tumor characteristic like ulceration. In addition, patient's age, tumor stage and lymph node involvement are also found to be significant factors affecting survival in patients with vulvar malignant melanoma. It has been observed that in patients with positive lymph nodes, the 5 -year disease specific survival is $24 \%$, compared with $68.3 \%$ for those with negative lymph nodes. ${ }^{5}$ Nevertheless, few authors have also documented that irrespective of the tumor stage the literature shows that 5-year survival rates are around $20-56 \% .{ }^{17}$

In order to assess the prognosis of patients with mucosal melanomas specifically, no universal staging system exists. In routine clinical practice, the Clark's tumor invasion level and Breslow tumor thickness classification for cutaneous melanoma are applied. The scale that is generally confirmed to be the most predictive of the overall survival for vulvar malignant melanoma is the 2002 American Joint Committee on Cancer Melanoma Staging System, which puts emphasis on the tumor invasion rather than tumor size, as in the FIGO classification of gynecological tumors, and therefore is of more validity in mucosal melanomas than the FIGO classification. ${ }^{3}$ Overall, the behavior of primary vulvar malignant melanomas and extragenital cutaneous melanomas appear to be similar. However, some studies have shown that the general prognosis of patients with vulvar malignant melanoma is worse than the cases with extragenital melanoma and invasive vulva carcinoma, showing a greater tendency towards locoregional and distant recurrence. ${ }^{18}$ This fact has been well-supported by various authors who have observed that in comparison with cutaneous $(80.8 \%)$ and ocular melanomas $(74.6 \%)$, vulvar malignant melanomas have the lowest 5-year survival rate, which ranges from $31.6 \%$ to $63 \%$, with an average of $44.6 \%$. It has also shown high recurrence rates of $63 \%$ and $67 \%$, which has been attributed to the inherent abnormality of the melanocytes rather than the surgical failure. ${ }^{19,20}$

\section{Conclusion}

Vulvar malignant melanoma is a rare neoplasm, with a poorer prognosis than cutaneous melanoma because of the tumor diversity, associated aggressiveness, its late detection, and failure of any effective therapy. Nevertheless, an early recognition of this entity can improve the patient management and survival rate. A multidisciplinary approach irrespective of patient's age comprising of a proper history, clinical examination, cytological, and histopathological correlation is advisable in such cases for clinching an accurate diagnosis as well as to avoid any diagnostic confusion and therapeutic delay. However, more insight is required to elucidate the etiopathogenesis of this extracutaneous melanoma, which is apparently not linked to UV radiation so that these researches on melanomagenesis can pave a way for new potential therapies in near future.

\section{Acknowledgments}

None.

\section{Conflicts of interest}

The authors declare there are no conflicts of interest.

\section{Funding}

None. 


\section{References}

1. Sturgeon SR, Brinton LA, Devesa SS, et al. In situ and invasive vulvar cancer incidence trends. Am J Obstet Gynecol. 1992;166(5):14821485 .

2. Chhabra S, Bhavani M, Deshpande A. Trends of vulvar cancer. J Obstet Gynaecol. 2014;34(2):165-168.

3. Jahnke A, Makovitzky J, Briese V. Primary melanoma of the female genital system: a report of 10 cases and review of the literature. Anticancer Res. 2005;25(3A):1567-1574

4. Trone JC, Guy JB, Mery B, et al. Melanomas of the female genital tract: state of the art. Bull Cancer. 2014;101(1):102-106.

5. Sugiyama VE, Chan JK, Shin JY, et al. Vulvar melanoma: a multivariable analysis of 644 patients. Obstet Gynecol. 2007;110(2 Pt 1):296-301.

6. Lemańska A, Banach P, Magnowska M, et al. Vulvar melanoma with urethral invasion and bladder metastases - a case report and review of the literature. Arch Med Sci. 2015;11(1):240-252.

7. Ragnarsson-Olding BK, Kanter-Lewensohn LR, Lagerlöf B, et al Malignant melanoma of the vulva in a nationwide, 25-year study of 219 Swedish females: clinical observations and histopathologic features. Cancer. 1999;86(7):1273-1284.

8. Moan J, Porojnicu AC, Dahlback A, et al. Where the sun does not shine: is sunshine protective against melanoma of the vulva? J Photochem Photobiol B. 2010;101(2):179-183.

9. Lundberg R, Brytting M, Dahlgren L, et al. Human herpes virus DNA is rarely detected in non-UV light-associated primary malignant melanomas of mucous membranes. Anticancer Res. 2006;26(5B):3627_ 3631 .

10. Giraud G, Ramqvist T, Ragnarsson-Olding B, et al. DNA from BK virus and $\mathrm{JC}$ virus and from $\mathrm{KI}, \mathrm{WU}$, and MC polyomaviruses as well as from simian virus 40 is not detected in non-UV-light-associated primary malignant melanomas of mucous membranes. $J$ Clin Microbiol. 2008;46(11):3595-3598.
11. Ragnarsson-Olding BK. Primary malignant melanoma of the vulva An aggressive tumor for modeling the genesis of Non-UV light-associated melanomas. Acta Oncol. 2004;43(5):421-435.

12. Singla S, Sharma S, Khambra P, et al. Cytodiagnosis of an Unsuspected Case of Metastatic Malignant Melanoma. Rec Adv Path Lab Med. 2016;2(1):2-3

13. Garg S, Sharma S. Metastatic malignant melanoma from an unknown primary presenting as an axillary mass. Asian Pac J Health Sci. 2018;5(1):25-27.

14. Errarhay S, Mamouni N, Mahmoud S, et al. Primary malignant melanoma of the female genital tract: unusual localization. JCR. 2013;3(1):169-175.

15. Stellato G, Lodice F, Casella G, et al. Primary malignant melanoma of the vagina: case report. Eur J Gynecol Oncol. 1998;19(2):186-188.

16. Nakagawa S, Koga K, Kugu K, et al. The evaluation of the sentinel node successfully conducted in a case of malignant melanoma of the vagina. Gynecol Oncol. 2002;86(3):387-389.

17. Bradgate MG, Rollason TP, McConkey CC, et al. Malignant melanoma of the vulva: a clinicopathological study of 50 women. $\mathrm{Br} J$ Obstet Gynaecol. 1990;97(2):124-133.

18. Janco JM, Markovic SN, Weaver AL, et al. Vulvar and vaginal melanoma: case series and review of current management options including neoadjuvant chemotherapy. Gynecol Oncol. 2013;129(3):533-537.

19. Verschraegen CF, Benjapibal M, Supakarapongkul W, et al. Vulvar melanoma at the M. D. Anderson Cancer Center: 25 years later. Int $J$ Gynecol Cancer. 2001;11(5):359-364.

20. Lotem M, Anteby S, Peretz T, et al. Mucosal melanoma of the female genital tract is a multifocal disorder. Gynecol Oncol. 2003;88(1):45-50. 